\title{
Synthesis, Characterization and DFT/TD-DFT Calculations of Highly Phosphorescent Blue Light-Emitting Anionic Iridium Complexes
}

Davide Di Censo, ${ }^{1}$ Simona Fantacci, ${ }^{2, *}$ Filippo De Angelis, ${ }^{2}$ Cedric Klein, ${ }^{1}$ Nick Evans, ${ }^{1} \mathrm{~K}$. Kalyanasundaram, ${ }^{1}$ Henk J. Bolink, ${ }^{3}$ Michael Grätzel ${ }^{1}$ and Mohammad K. Nazeeruddin ${ }^{1, *}$

${ }^{1}$ Laboratory for Photonics and Interfaces, Station 6, Institute of Chemical Sciences and Engineering, School of basic Sciences, Swiss Federal Institute of Technology, CH - 1015 Lausanne, Switzerland

${ }^{2}$ Istituto CNR di Scienze e Tecnologie Molecolari (ISTM-CNR), c/o Dipartimento di Chimica, Università di Perugia, I-06123, Perugia, Italy.

${ }^{3}$ Institute of Molecular Science, University of Valencia ,P.O. Box 22085 ES-46071 Valencia, Spain,

E-Mail: MdKhaja.Nazeeruddin@epfl.ch, simona@thch.unipg.it 


\section{Supporting Information}

Table S1. Optimized geometrical parameters of complexes 1-5.

\begin{tabular}{|c|c|c|c|c|c|c|c|c|c|c|c|}
\hline & 1 (X-ray) & \multicolumn{2}{|c|}{1} & \multicolumn{2}{|c|}{2} & \multicolumn{2}{|c|}{3} & \multicolumn{2}{|c|}{4} & \multicolumn{2}{|c|}{5} \\
\hline Parameter & $\mathrm{S}_{0}$ & $\mathrm{~S}_{0}$ & $\mathrm{~T}_{1}$ & $\mathrm{~S}_{0}$ & $\mathrm{~T}_{1}$ & $\mathrm{~S}_{0}$ & $\mathrm{~T}_{1}$ & $\mathrm{~S}_{0}$ & $\mathrm{~T}_{1}$ & $\mathrm{~S}_{0}$ & $\mathrm{~T}_{1}$ \\
\hline 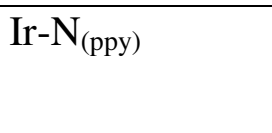 & $\begin{array}{l}2.061(4) \\
2.063(4)\end{array}$ & 2.046 & 2.056 & 2.045 & 2.060 & 2.048 & 2.060 & 2.050 & 2.056 & 2.052 & 2.065 \\
\hline Ir-C $C_{(\text {ppy) }}$ & $\begin{array}{l}2.055(5) \\
2.059(5)\end{array}$ & 2.051 & 2.031 & 2.051 & 2.027 & 2.050 & 2.040 & 2.048 & 2.041 & 2.068 & 2.040 \\
\hline $\operatorname{Ir}-\mathrm{C}_{(\mathrm{CN})}$ & $\begin{array}{l}2.066(5) \\
2.079(5)\end{array}$ & 2.060 & 2.076 & 2.057 & 2.077 & 2.056 & 2.056 & 2.054 & 2.058 & 2.046 & 2.057 \\
\hline $\mathrm{C}_{(\mathrm{ppy})}-\mathrm{C}_{(\mathrm{ppy})}$ & $\begin{array}{l}1.457(7) \\
1.463(6)\end{array}$ & 1.451 & 1.436 & 1.456 & 1.437 & 1.450 & 1.434 & 1.455 & 1.432 & 1.457 & 1.435 \\
\hline$<\mathrm{N}_{(\mathrm{ppy})}-\mathrm{Ir}-\mathrm{C}_{(\mathrm{ppy})}$ & $\begin{array}{l}79.85(17) \\
79.41(17)\end{array}$ & 79.6 & 80.8 & 79.3 & 80.8 & 79.7 & 80.6 & 79.4 & 80.5 & 78.9 & 80.0 \\
\hline$<\mathrm{N}_{(\mathrm{ppy})}-\mathrm{Ir}-\mathrm{N}_{(\mathrm{ppy})}$ & $170.01(14)$ & 171.9 & 173.1 & 171.3 & 173.2 & 171.8 & 172.6 & 171.5 & 172.2 & 174.5 & 175.8 \\
\hline$<\mathrm{C}_{(\mathrm{ppy})}-\operatorname{Ir}-\mathrm{C}_{(\mathrm{ppy})}$ & $86.63(17)$ & 91.7 & 92.4 & 91.7 & 95.0 & 91.7 & 91.4 & 91.9 & 91.3 & 91.6 & 96.6 \\
\hline$<\mathrm{C}_{(\mathrm{CN})}-\mathrm{Ir}-\mathrm{C}_{(\mathrm{CN})}$ & $89.92(19)$ & 89.7 & 88.1 & 88.5 & 85.3 & 89.0 & 89.1 & 89.0 & 88.9 & 89.8 & 84.9 \\
\hline
\end{tabular}

\section{DFT results vs. electrochemical data}

In complex $\mathbf{2}$, the para-substitution of pyridine by dimethylamino group leads to a destabilization of both the HOMOs and LUMOs. Compared to the $\mathbf{1}$ reference, the $\mathbf{2}$ HOMO is destabilized by $0.215 \mathrm{eV}$ while the LUMO is destabilized by $0.317 \mathrm{eV}$, as a result of the increase donation from the dimethylamino-substituted ppy. The net effect is a small opening of the HOMOLUMO gap, which increases from 3.889 to $3.990 \mathrm{eV}$. The calculated HOMO/LUMO shifts quantitatively correlate with the experimental redox potentials, showing an upshift of the oxidation and reduction potentials of 0.220 and $0.310 \mathrm{~V}$, respectively. In complex $\mathbf{3}$, the presence of fluorine substituents in 2,4 positions of phenyl rings does stabilizes the anionic complex resulting into an overall stabilization of all the HOMOs and LUMOs with respect to the orbitals of complex $\mathbf{1}$. In particular, the HOMO (LUMO) is stabilized by $0.479(0.306) \mathrm{eV}$, which is consistent with the 
experimental trend of oxidation/reduction potentials. The calculated HOMO-LUMO gap is 4.062 $\mathrm{eV}$, i.e. $0.173 \mathrm{eV}$ larger than in $\mathbf{1}$, which stems from the different stabilization of the HOMO and LUMO. The HOMOs of complexes $\mathbf{4}$ and $\mathbf{5}$ result stabilized with respect to both those of $\mathbf{1}$ and $\mathbf{2}$, which have no fluorine substituents. Indeed, the electron distribution of the first HOMOs resembles to that of complex $\mathbf{2}$ HOMOs, a part that the $\mathbf{2}$ HOMO corresponds to HOMO-2 in both $\mathbf{4}$ and $\mathbf{5}$, therefore resulting particularly stabilized by the presence of $\mathrm{F}$ atoms.

The two LUMOs of $\mathbf{4}$ and $\mathbf{5}$ complexes are destabilized with respect to the LUMO of complex 1, as a result of the electron donating and electron withdrawing effect of the dimethylamino and fluoride ligands, respectively. Interestingly, the LUMO+2/LUMO+3 and LUMO+4/LUMO+5 couples, which are essentially localized on the pyridine and phenyl groups, respectively, feel differently the effect of the substituents. Indeed, in both $\mathbf{4}$ and $\mathbf{5}$, the LUMO+2 and LUMO+3 are destabilized with respect to the same couple of orbitals of $\mathbf{1}$, and lie at similar energies as the LUMO+2 and LUMO+3 of 2. Complex 4 shows the largest HOMO-LUMO gap among the investigated species $(4.137 \mathrm{eV})$ as a results of the concomitant HOMO stabilization and LUMO destabilization. In the isomeric complex 5, the HOMO-LUMO gap is calculated to be 4.060 $\mathrm{eV}$, the decreased value compared to that of $\mathbf{4}$ being related to smaller HOMO (LUMO) stabilization (destabilization). For 4, our calculated molecular orbitals are consistent with experimental redox potentials, showing a down-shift (up-shift) of the oxidation (reduction) potentials compared to $\mathbf{1}$. We notice, moreover, that comparing $\mathbf{4}$ and $\mathbf{5}$, our calculations confirm the experimental trend of redox potentials, even though for complex $\mathbf{5}$, compared to $\mathbf{1}$, our calculations would predict an anodic shift of the oxidation potential, while experimentally a slight cathodic shift is observed. The only difference which can justify the electrochemical behavior of 4 and 5 complexes comes from the charge density distribution of LUMO and LUMO+1 of the two complexes. In complex 2 , the LUMO/LUMO+1 couple shows electron charge distribution in both the meta and ortho positions of phenyl ring. When hydrogen atoms are replaced by fluorides, together to the main stabilization effect on the LUMO/LUMO+1 due to the electron attractor 
character of these substituent, we noticed a minor effect related to the position of fluoride. In fact, fluorides in meta and ortho positions cause additional $\pi$ interactions of antibonding character, which are missing in the para position, this effect reflects on a slight stabilization of LUMO/LUMO+1 of complex 5 with respect to those of complex $\mathbf{4}$, and could provide a possible explanation for the change in the reduction potentials of the two complexes.

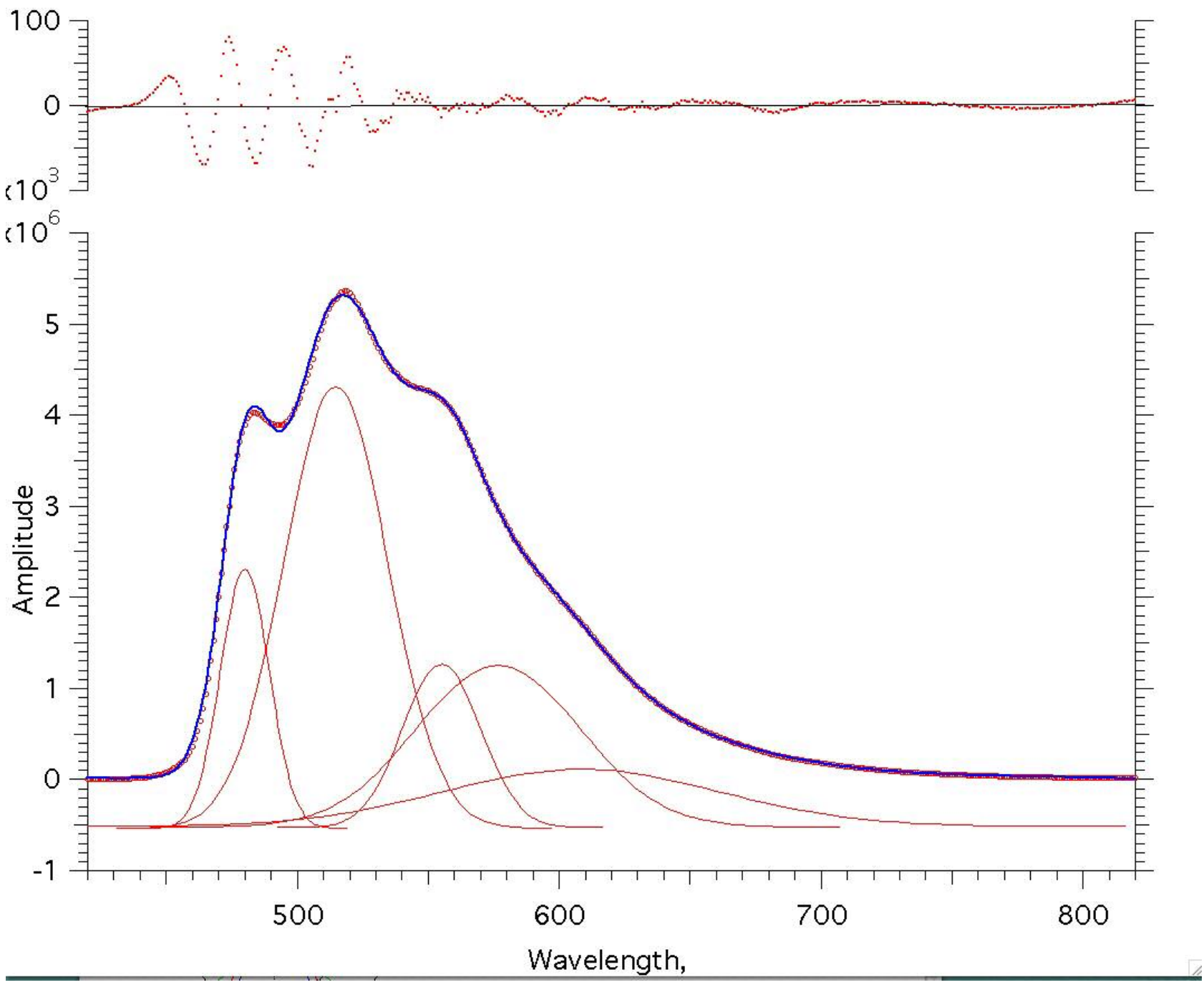

Figure SI-1. Emission spectra of complex 4 fitted with using a multigaussion Igor pro. 